\title{
Knowledge and Practice Discordance in Treating Urinary Tract Infections
}

David W. Bauer, MD | Julie Adkison, PharmD | Heather Hamilton, MD

PRIMER. 2018;2:26.

Published: 11/19/2018 | DOI: 10.22454/PRiMER.2018.742456

\section{Abstract}

Background and Objectives: Urinary tract infections (UTIs) are a common condition in women. Guidelines have been published on the diagnosis and management of such infections with the twin goals of improved patient safety and avoiding unnecessary health care costs. In this study we evaluated both physicians' knowledge of these guidelines, and whether that knowledge translates to their clinical practice.

Methods: We performed a retrospective chart review of UTI management before and after an educational intervention in a family medicine residency. Two control sites were also included. We also assessed physicians' knowledge of current UTI management guidelines using an anonymous quiz.

Results: At the intervention site, guideline adherence improved following the educational session. Inappropriate culture use decreased from $88 \%$ to $68 \%(P<.005)$ while first-line antibiotic use increased from $68 \%$ to $92 \%(P<.005)$. No such benefits were seen at the control sites. The control and intervention site providers did not differ in knowledge of antibiotic choice on the quiz ( $86 \%$ and $88 \%$ correct respectively, $P=.85$ ), but all control providers felt routine cultures were appropriate, whereas only $38 \%$ of the intervention providers $\operatorname{did}(P<.05)$.

Conclusions: A simple educational intervention made an impact on the management of UTI. Our results also highlighted that knowledge and practice are not always aligned.

\section{Introduction}

In 2010 the Infectious Diseases Society of America (IDSA) published guidelines for the treatment of acute uncomplicated cystitis. ${ }^{1}$ Adequate antibiotic therapy is correlated with improved clinical outcomes, decreased antimicrobial resistance, and lower costs. ${ }^{2}$ The IDSA guideline provides specific recommendations for optimal firstline therapy for uncomplicated urinary tract infection (UTI) that include trimethoprim-sulfamethoxazole, nitrofurantoin, or fosfomycin (although this agent is rarely used). Fluoroquinolones should only be considered when first-line options cannot be used.

Patient history, symptoms, and urinalysis should be the primary criteria to make a diagnosis of uncomplicated UTI. ${ }^{3}$ Numerous practice guidelines have recommended against routine urine cultures in uncomplicated UTI for cost reasons. ${ }^{4-6}$ Despite these recommendations, the use of urine cultures in UTI diagnosis remains high. ${ }^{7-9}$

We hypothesized our UTI diagnosis and treatment were suboptimal in light of guideline standards. Our study sought to assess provider knowledge of IDSA treatment guidelines, compliance with urine cultures and prescribing, and whether a simple educational intervention could improve adherence. 


\section{Methods}

The University of Texas Institutional Review Board approved the study. The intervention site was a family medicine residency with 42 residents, 12 faculty, and one physician assistant. The control sites were two clinics staffed by two family physicians and five nurse practitioners. All three sites were located in the same city. The study consisted of two components: a retrospective chart review, and a knowledge assessment.

\section{Chart Review}

A random sample of female patients between the ages of 18 and 65 years with dysuria were included in a retrospective chart review. Patients were excluded if they had known lower or upper tract malignancy, were pregnant, had signs of systemic infection, recent instrumentation, flank tenderness, vaginal discharge, renal dysfunction, a ureteral stent or a UTI in the prior 3 months. All providers were included.

Data collected included age, urinalysis findings, urine culture results, antibiotic choice, and antibiotic allergies or contraindications. The first set of data was collected from visits between January 2016 and August 2016, prior to an educational intervention that took place in September, 2016. The second set of data was collected after the intervention, between October 2016 and March 2017.

\section{Educational Intervention and Knowledge Quiz}

The educational intervention delivered was a noon conference on best practices in diagnosing and managing UTI, followed by a summary highlighting the important points of the IDSA guideline emailed to providers. Only providers at the intervention site received this material. We used Qualtrics survey software to send an anonymous quiz to providers to evaluate guideline knowledge. Data from the chart review were analyzed using the Analyse-it software package, version 4.60.1.

\section{Results}

\section{Chart Review}

Two random samples of charts were reviewed at each site. The first were patients seen prior to the educational intervention, and the second were patients seen postintervention. At the intervention site, 124 patients were included, and 91 were included at the control sites. Sample size did not allow analysis of effect size by provider type.

\section{Use of Culture}

A binomial logistic regression analysis was performed using independent variables of time (before vs after the intervention) and location (control site vs intervention site), and a dependent variable of likelihood of ordering a culture. This analysis demonstrated no significant change in culture ordering in the control group ( $97 \%$ before, $94 \%$ after), but a significant decrease in inappropriate culture ordering in the intervention group ( $88 \%$ to $68 \%, P<.005$; Figure 1).

\section{Antibiotic Choice}

A similar analysis was performed using the same independent variables and a dependent variable of empiric antibiotic choice (first line or not). Again, a binomial logistic regression analysis revealed the intervention site (68\% to $92 \%, P<.005)$ saw an increase in first-line antibiotic use following the intervention, but the control site did not $(42 \%$ to $41 \%$, NS; Figure 2).

Empiric antibiotics prescribed fell into four classes: $\beta$-lactams, fluoroquinolones, nitrofurantoin, and trimethoprimsulfamethoxazole (no prescriptions for fosfomycin were found). While the control groups' prescribing pattern changed somewhat between the two intervals, the proportion of first-line agents did not $(P=0.96)$. Rather, there was an increase in nitrofurantoin and corresponding decrease in trimethoprim-sulfamethoxazole. In other words, one first-line agent was replaced with another. In contrast, there was a highly significant increase in first-line treatment at 
the intervention site $(P<.005)$. Here the change reflects a decrease in fluoroquinolones and corresponding increase in nitrofurantoin (Figure 3).

\section{Knowledge Quiz}

Forty-one providers completed the knowledge quiz. Seven were from the control sites, and the remainder were from the intervention site. The two groups did not differ in selecting the correct antibiotic for uncomplicated UTI ( $86 \%$ in the control group and $88 \%$ in the intervention group, $\chi^{2} P=.85$ ). The intervention group was significantly better than the control group in recognizing that a culture is not indicated $(0 \%$ correct for the control group and $62 \%$ for the intervention group, $\left.\chi^{2} P<.005\right)$.

\section{Conclusions}

The results of this study reveal two important findings. First, a simple educational intervention improved UTI management. Second, providers' knowledge of the guidelines in a hypothetical scenario was better than actual patient care. This suggests that guideline nonadherence is more complex than lack of knowledge.

Although UTI guidelines have been published in several countries, adherence remains low. Multiple studies show overordering cultures and prescribing second-line antibiotics. Compliance rates for ordering preferred antibiotics ranges from $17 \%-64 \%$, well short of the IDSA goal of $80 \% .{ }^{10-14}$ Barriers include lack of guideline awareness, clinical decision making based on physician beliefs and habits, and cultural norms. ${ }^{15-17}$

Efforts previously reported to improve UTI guideline adherence have been multifaceted and time intensive. ${ }^{18,19}$ Using our straightforward intervention of an educational lecture with one follow-up summary email, we showed an absolute increase of $24 \%$ in appropriate antibiotic selection. Replicating our inexpensive and simple educational effort may be feasible across a variety of practice settings and diagnoses.

Interventions aimed at improving diagnostic confidence could decrease culture ordering rates. Mclsaac and colleagues ${ }^{20}$ concluded that a three-item clinical decision aid for women with symptoms of acute cystitis could lead to clinically important reductions in total and unnecessary antibiotic use and culture testing.

Study limitations include data collection at a single site, without a control residency practice to compare our findings (although we did have nonresidency control sites). This leads to uncertainty in ability to generalize results. The knowledge quiz was anonymous; therefore, we are unable to correlate an individual's performance on the quiz with his or her clinical practice. We did not track attendance for the session where the educational information was presented, and don't know which physicians received the training. However, all providers at the intervention site received an electronic summary of the training points. We were not able to separately analyze resident vs faculty changes in practice due to sample size limitations. No ambulatory antibiogram exists in our area, so we do not know the local resistance rates, which would alter guideline recommendations. Finally, the duration of the effect of the educational intervention is unknown.

We have demonstrated efficacy of an educational intervention in conferring adherence to UTI practice guidelines. We have also demonstrated a discordance between physicians' knowledge of managing patients with UTI and actual care. We find it encouraging that a relatively simple educational intervention appeared to lead to significant improvement in patient care. Educational interventions targeted toward areas of knowledge deficits may be an efficient way to improve guideline adherence. A follow-up study looking at the key criteria of guideline adherence for 1 year or more postintervention would help clarify the longevity of practice change.

\section{Tables and Figures}


Figure 1: Percent of Patients for Whom No Culture Was Ordered at the Control and Intervention Site, Before and After the Intervention

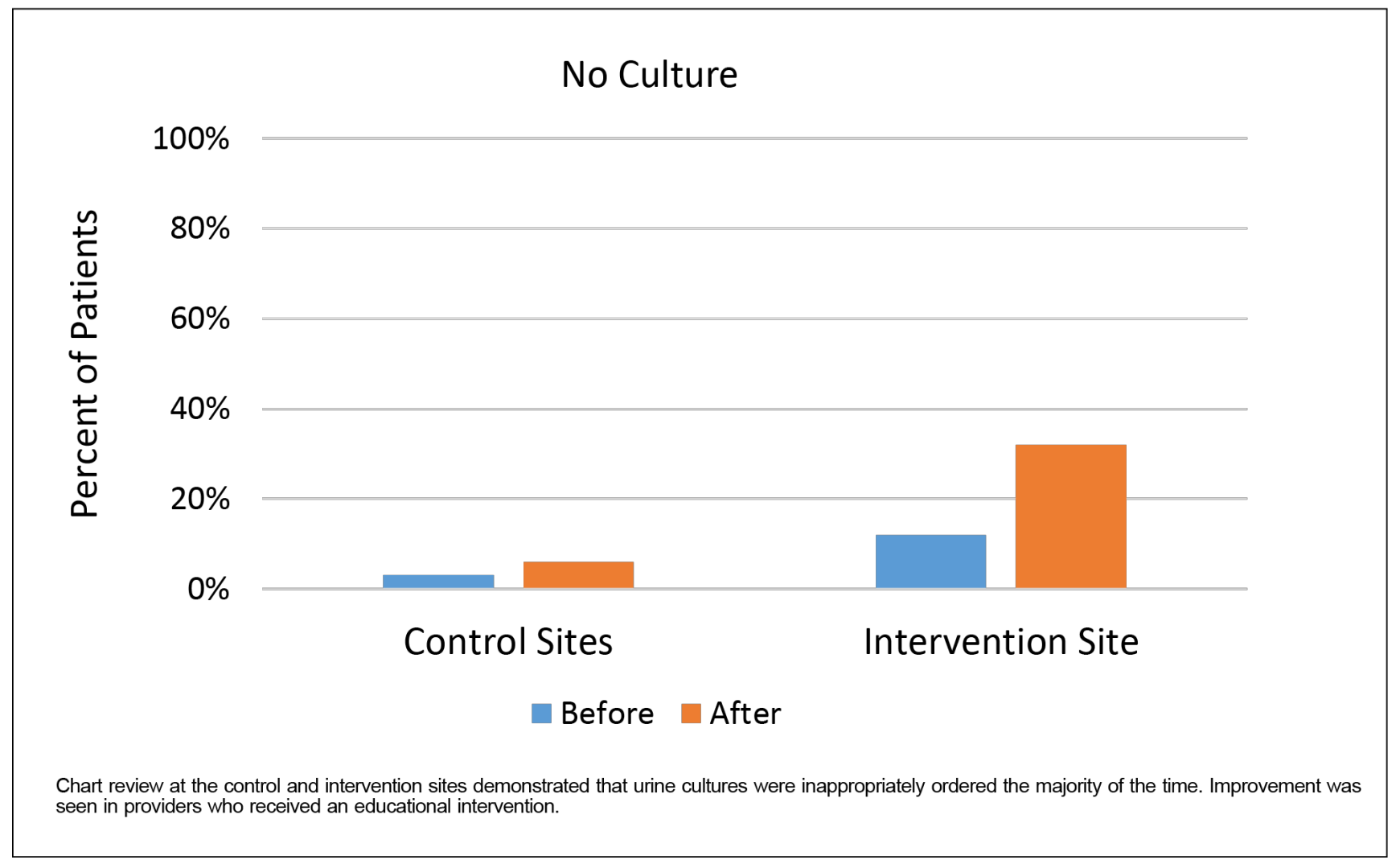

Figure 2: Percent of Patients Receiving Empiric First-Line Antibiotics at the Control and Intervention Site, Before and After the Intervention

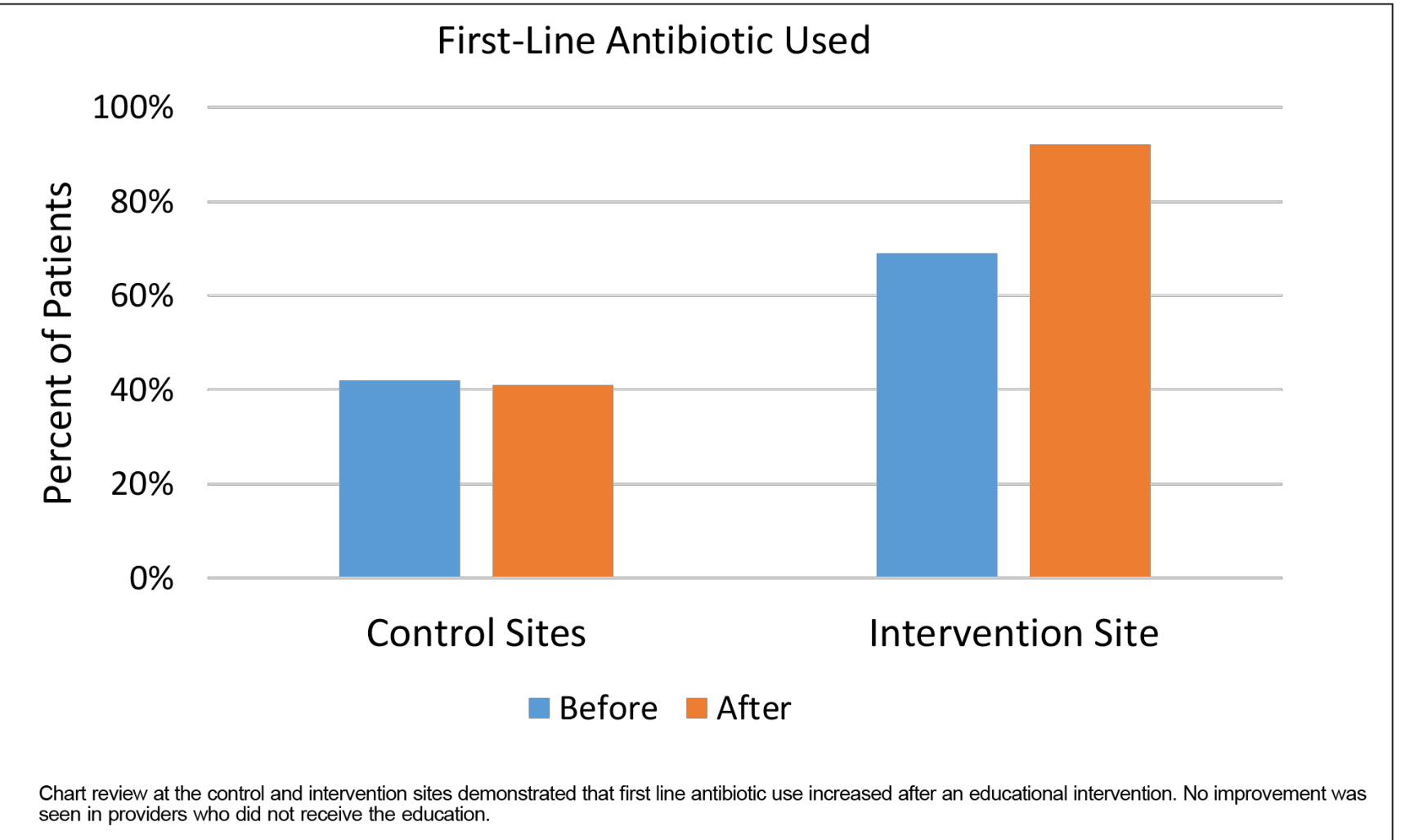


Figure 3: Frequency of Antibiotic Choice at the Control and Intervention Sites, Before and After the Intervention

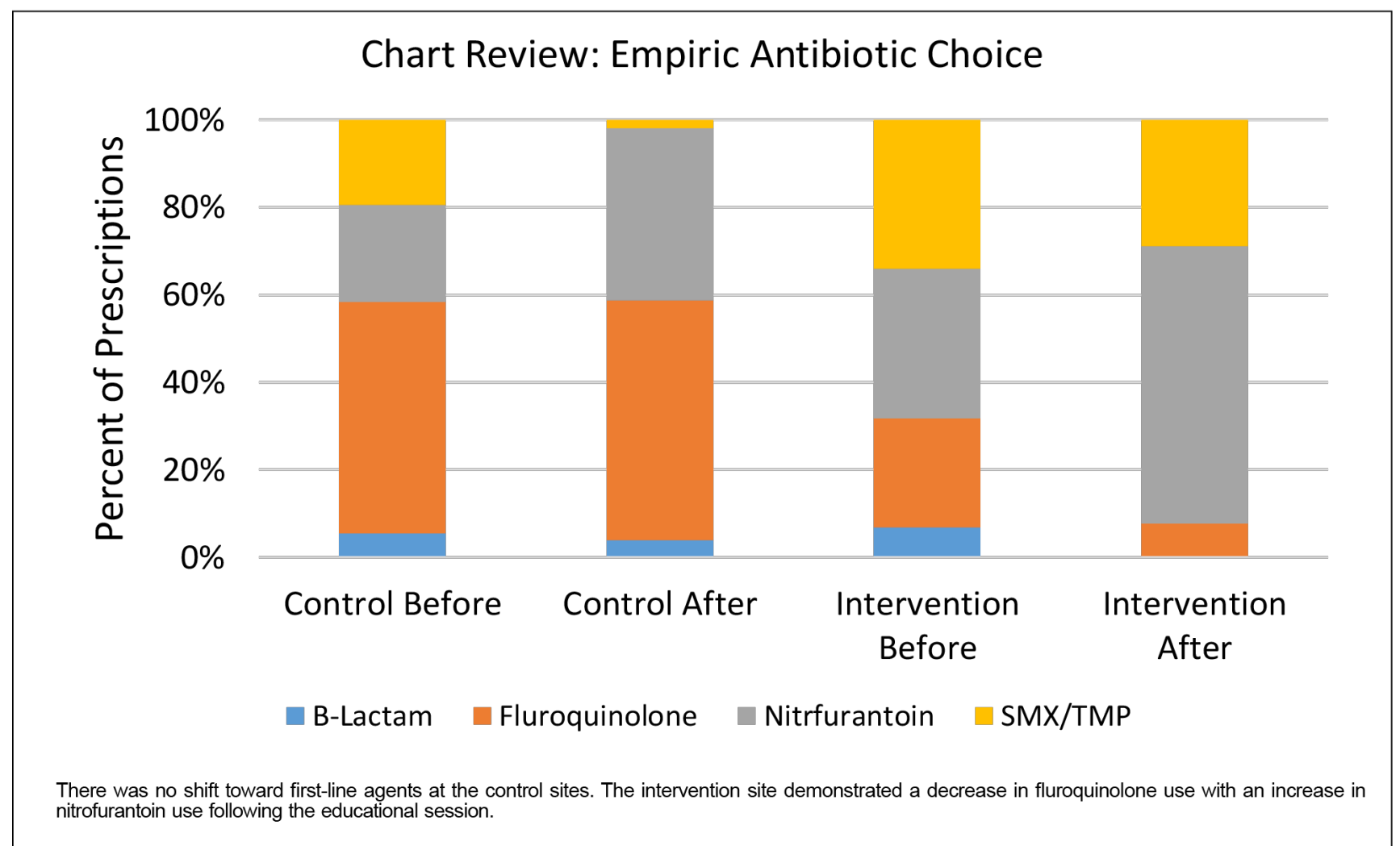

\section{Acknowledgments}

The authors wish to thank Monica Kalra, DO, for her advice on statistical analysis, and Bich-May Nguyen, MD, for her review and comments on an earlier version of this paper.

\section{Corresponding Author}

David W. Bauer, MD

Memorial Family Medicine Residency Program, 14023 Southwest Freeway, Sugar Land, TX 77478. 281-325-4250.

Fax: 281-325-4292.

david.bauer@memorialhermann.org

\section{Author Affiliations}

David W. Bauer, MD - Memorial Family Medicine Residency Program, Sugar Land, TX

Julie Adkison, PharmD - Memorial Family Medicine Residency Program, Sugar Land, TX

Heather Hamilton, MD - Memorial Hermann Medical Group, Houston, TX

\section{References}

1. Gupta K, Hooton TM, Naber KG, et al; Infectious Diseases Society of America; European Society for Microbiology and Infectious Diseases. International clinical practice guidelines for the treatment of acute uncomplicated cystitis and pyelonephritis in women: A 2010 update by the Infectious Diseases Society of America and the European Society for Microbiology and Infectious Diseases. Clin Infect Dis. 2011;52(5):e103e120. https://doi.org/10.1093/cid/ciq257

2. Warren JW, Abrutyn E, Hebel JR, Johnson JR, Schaeffer AJ, Stamm WE; Infectious Diseases Society of America (IDSA). Guidelines for antimicrobial treatment of uncomplicated acute bacterial cystitis and acute 
pyelonephritis in women. Clin Infect Dis. 1999;29(4):745-758. https://doi.org/10.1086/520427

3. Michels TC, Sands JE. Dysuria: evaluation and differential diagnosis in adults. Am Fam Physician. 2015;92(9):778-786.

4. Fenwick EA, Briggs $\mathrm{AH}$, Hawke $\mathrm{Cl}$. Management of urinary tract infection in general practice: a costeffectiveness analysis. Br J Gen Pract. 2000;50(457):635-639.

5. Hooton TM. Practice guidelines for urinary tract infection in the era of managed care. Int $\mathrm{J}$ Antimicrob Agents. 1999; 11(3-4):2 41-45.

6. Turner D, Little P, Raftery J, et al; UTIS group. Cost effectiveness of management strategies for urinary tract infections: results from randomised controlled trial. BMJ. 2010;340(feb05 1):c346. https://doi.org/10.1136 /bmj.c346

7. Hummers-Pradier E, Ohse AM, Koch M, Heizmann WR, Kochen MM. Management of urinary tract infections in female general practice patients. Fam Pract. 2005;22(1):71-77. https://doi.org/10.1093/fampra/cmh720

8. Johnson JD, O'Mara HM, Durtschi HF, Kopjar B. Do urine cultures for urinary tract infections decrease followup visits? J Am Board Fam Med. 2011;24(6):647-655. https://doi.org/10.3122/jabfm.2011.06.100299

9. Wigton RS, Longenecker JC, Bryan TJ, Parenti C, Flach SD, Tape TG. Variation by specialty in the treatment of urinary tract infection in women. J Gen Intern Med. 1999;14(8):491-494. https://doi.org/10.1046 /j.1525-1497.1999.05398.x

10. Llor C, Rabanaque G, López A, Cots JM. The adherence of GPs to guidelines for the diagnosis and treatment of lower urinary tract infections in women is poor. Fam Pract. 2011;28(3):294-299. https://doi.org/10.1093 /fampra/cmq107

11. Kahan E, Kahan NR, Chinitz DP. Urinary tract infection in women-physician's preferences for treatment and adherence to guidelines: a national drug utilization study in a managed care setting. Eur $\mathrm{J}$ Clin Pharmacol. 2003;59(8-9):663-668. https://doi.org/10.1007/s00228-003-0673-4

12. Sigler M, Leal JE, Bliven K, Cogdill B, Thompson A. Assessment of appropriate antibiotic prescribing for urinary tract infections in an internal medicine clinic. South Med J. 2015;108(5):300-304. https://doi.org /10.14423/SMJ.0000000000000278

13. Grover ML, Bracamonte JD, Kanodia AK, et al. Assessing adherence to evidence-based guidelines for the diagnosis and management of uncomplicated urinary tract infection. Mayo Clin Proc. 2007;82(2):181-185. https://doi.org/10.1016/S0025-6196(11)60996-8

14. Mclsaac WJ, Prakash P, Ross $S$. The management of acute uncomplicated cystitis in adult women by family physicians in Canada. Can J Infect Dis Med Microbiol. 2008;19(4):287-293. https://doi.org/10.1155 /2008/404939

15. Taur Y, Smith MA. Adherence to the Infectious Diseases Society of America guidelines in the treatment of uncomplicated urinary tract infection. Clin Infect Dis. 2007;44(6):769-774. https://doi.org/10.1086/511866

16. Charani E, Edwards R, Sevdalis N, et al. Behavior change strategies to influence antimicrobial prescribing in acute care: a systematic review. Clin Infect Dis. 2011;53(7):651-662. https://doi.org/10.1093/cid/cir445

17. Kaplan SH, Griffith JL, Price LL, Pawlson LG, Greenfield S. Improving the reliability of physician performance assessment: identifying the "physician effect" on quality and creating composite measures. Med Care. 2009;47(4):378-387. https://doi.org/10.1097/MLR.0b013e31818dce07

18. Vellinga A, Galvin S, Duane $S$, et al. Intervention to improve the quality of antimicrobial prescribing for urinary tract infection: a cluster randomized trial. CMAJ. 2016;188(2):108-115. https://doi.org/10.1503/cmaj.150601

19. Willems L, Denckens P, Philips H, Henriquez R, Remmen R. Can we improve adherence to guidelines for the treatment of lower urinary tract infection? A simple, multifaceted intervention in out-of-hours services. J Antimicrob Chemother. 2012;67(12):2997-3000. https://doi.org/10.1093/jac/dks336

20. Mclsaac WJ, Moineddin R, Gágyor I, Mazzulli T. External validation study of a clinical decision aid to reduce unnecessary antibiotic prescriptions in women with acute cystitis. BMC Fam Pract. 2017;18(1):89.

https://doi.org/10.1186/s12875-017-0660-y

Copyright $(2018$ by the Society of Teachers of Family Medicine 